\title{
Improved Protection from Velogenic Newcastle Disease Virus Challenge Following Multiple Immunizations With Plasmid DNA Encoding for F And HN Genes
}

\begin{abstract}
Specific-pathogen free (SPF) chicken were inoculated with the plasmid combination and challenged with velogenic NDV. The antibody level against NVD was measured using commercial enzyme linked immunosorbent assay (ELISA). In the first immunization regimen, SPF chickens inoculated twice with NDV-F or NDV-HN constructs elicited antibody responses 1 week after the second injection. However, the levels of the antibody were low and did not confer significant protection from the lethal challenge. In addition, admininistration of the plasmid constructs with Freund's adjuvant did not improve the level of protection. In the second immunization regimen, chickens inoculsted trice with the plasmid constructs emulsified with Freund"s adjuvant induced significant antibody titers after the third injection. Three out of nine $(33.3 \%)$ chickensvaccinated with pEGFP-HN, five of ten (50.0\%) chicken vaccinated with pEGFP-F and nine of ten $(90.0 \%)$ chicken vaccinated with combined pEGFP-F and pEGFP-HN were protected from the challenge. No significant differences in the levels of protection were observed when the chicken were vaccinated with linearized pEGFP-F. the results suggested that more than two injections with both F and HN encoding plasmid DNA were required to induce level of antibodies for protection against velogenic NDV in chickens.
\end{abstract}

